\title{
USO DE MESOCARPO DE MARACUJÁ-AMARELO COMO BIOSSORVENTE PARA REMOÇÃO DE Cr(VI) DE SOLUÇÕES AQUOSAS
}

\author{
Y. de J. COSTA ${ }^{1}$, T. F. SOUZA ${ }^{1}$, E. N. dos REIS ${ }^{1}$ e E. de J. SANTOS ${ }^{1}$ \\ ${ }^{1}$ Universidade Federal de Sergipe, Departamento de Engenharia Química \\ E-mail para contato: yasmincosta.ba@gmail.com
}

\begin{abstract}
RESUMO - Este estudo objetivou avaliar a potencialidade do mesocarpo de maracujá (Passiflora edulis) como biossorvente no tratamento de soluções aquosas com $\mathrm{Cr}(\mathrm{VI})$. O biomaterial foi obtido através de tratamento térmico a 400 ${ }^{\circ} \mathrm{C}$. A determinação de $\mathrm{Cr}$ (VI) foi feita por espectrofotometria UV-visível através da complexação com 1,5-difenilcarbazida, medindo a absorbância no comprimento de onda de $540 \mathrm{~nm}$. O tempo de contato necessário para atingir o equilíbrio foi 3 horas. Os dados de equilíbrio químico foram analisados usando os modelos de Langmuir e Freundlich e os parâmetros foram determinados através de Programação Matemática Não-Linear. O processo de biossorção de $\mathrm{Cr}(\mathrm{VI})$ sobre o mesocarpo de maracujá foi melhor representado pelo modelo de Freundlich. Os resultados mostraram que o biossorvente removeu em média 75\% de $\mathrm{Cr}(\mathrm{VI})$ em 180 minutos, possuindo capacidade máxima de biossorção de 13,0 $\mathrm{mg} \mathrm{g}^{-1}$.
\end{abstract}

\section{INTRODUÇÃO}

Um dos fatores que afetam a qualidade da água e poluem o meio ambiente é o lançamento de resíduos aquosos que contém metais pesados, a exemplo do $\mathrm{Cr}(\mathrm{VI})$ proveniente geralmente de indústrias de extração de minério, fábricas de cerveja e destilarias, indústrias de plástico, companhias de eletricidade, dentre outras (Pina, 2011). O Cr(VI) é tóxico e carcinogênico por causa do elevado potencial de oxidação e por sua elevada capacidade de penetrar em membranas biológicas, sendo que a excessiva exposição e inalação desse composto pode causar várias doenças.

Existem várias tecnologias para a remoção de metais pesados do meio ambiente (Conceição et al., 2014), sendo que as tecnologias convencionais apresentam custos demasiado elevados, o que tem levado ao aparecimento de novas tecnologias e métodos de redução/eliminação dos poluentes. Uma dessas alternativas é a biossorção que consiste na remoção de metais pesados dos efluentes utilizando materiais de origem biológica. Assim sendo, o mesocarpo de maracujá é um resíduo da agroindústria utilizado como biossorvente para remoção de $\mathrm{Cr}(\mathrm{VI})$ de efluentes industriais, tendo este trabalho caráter estabelecido pela sustentabilidade, que é o aproveitamento dos resíduos gerados pelos processos químicos.

No desenvolvimento de sistemas de adsorção para a remoção de um determinado adsorbato, é importante descrever os dados de equilíbrio através de um modelo matemático. A descrição do equilíbrio químico é importante para estabelecer parâmetros de capacidade de 
adsorção e de espontaneidade do processo. A forma das isotermas também é a primeira ferramenta experimental para conhecer o tipo de interação entre o adsorbato e o adsorvente. Neste estudo, foram utilizadas as isotermas de Langmuir e Freundlich.

O modelo de Langmuir assume que a superfície do adsorvente é completamente homogênea e que um número limitado de sítios do adsorvente é ocupado pelo soluto. A expressão do modelo de Langmuir é representado pela Equação 1.

$$
Q_{e}=\frac{k_{L} C_{e} Q_{\max }}{1+k_{L} C_{e}}
$$

em que $\mathrm{Q}_{\max }\left(\mathrm{mg} \mathrm{g}^{-1}\right)$ e $\mathrm{k}_{\mathrm{L}}\left(\mathrm{L} \mathrm{mg}^{-1}\right)$ são constantes relacionadas com a capacidade de adsorção máxima e a energia de adsorção máxima, respectivamente.

As características essenciais da isoterma de Langmuir podem ser explicadas em termos do fator de separação adimensional constante, $\mathrm{R}_{\mathrm{L}}$, dado pela Equação 2, na qual $\mathrm{k}_{\mathrm{L}} \mathrm{e}$ a constante de Langmuir e $\mathrm{C}_{\mathrm{o}}\left(\mathrm{mg} \mathrm{L}^{-1}\right)$ é a concentração inicial de $\mathrm{Cr}(\mathrm{VI})$. De acordo com o valor do fator de separação $\mathrm{R}_{\mathrm{L}}$ que é um número positivo cuja magnitude determina a viabilidade do processo de adsorção, existem os seguintes tipos de adsorção: (1) adsorção favorável, $0<\mathrm{R}_{\mathrm{L}}<1$; (2) adsorção desfavorável, $\mathrm{R}_{\mathrm{L}}>1$; (3) adsorção linear, $\mathrm{R}_{\mathrm{L}}=1$ e (4) adsorção irreversível, $\mathrm{R}_{\mathrm{L}}=0$.

$$
R_{L}=\frac{1}{1+k_{L} C_{o}}
$$

O modelo de Freundlich foi obtido empiricamente e tem a forma da Equação 3, apresentando bons resultados em superfícies heterogêneas como os carvões ativados.

$$
Q_{e}=k_{F} C_{e}^{1 / n}
$$

em que $\mathrm{Q}_{\mathrm{e}}$ é a quantidade do adsorbato adsorvida no equilíbrio $\left(\mathrm{mg} \mathrm{g}^{-1}\right), \mathrm{C}_{\mathrm{e}}$ é a concentração do adsorbato no equilíbrio $\left(\mathrm{mg} \mathrm{L}^{-1}\right)$ e $\mathrm{k}_{\mathrm{F}}\left[\left(\mathrm{mg} \mathrm{g}^{-1}\right)\left(\mathrm{L} \mathrm{mg}^{-1}\right)^{1 / n}\right]$ e $\mathrm{n}$ são as constantes de Freundlich. $\mathrm{k}_{\mathrm{F}}$ representa a capacidade de adsorção e $\mathrm{n}$ representa a intensidade do processo de adsorção.

\section{MATERIAIS E MÉTODOS}

\subsection{Preparação do Biossorvente}

O maracujá-amarelo in natura foi coletado na feira de Nossa Senhora do Socorro, Sergipe, em setembro de 2014. Em seguida, foi lavado com água no Laboratório de Química Industrial da Universidade Federal de Sergipe. O mesocarpo foi, então, devidamente retirado do maracujá, como mostra a Figura 1. O tratamento térmico consistiu em aquecimento em mufla (GP Científica) a $400{ }^{\circ} \mathrm{C}$ durante 30 minutos, sendo posteriormente triturado num processador de alimentos. A granulometria do biossorvente foi feita utilizando peneiras de 4, 9, 12, 32, 100 e 200 mesh e classificador granulométrico da Bertel. Utilizou-se o pó com granulometria de 100 mesh nas análises deste estudo. 


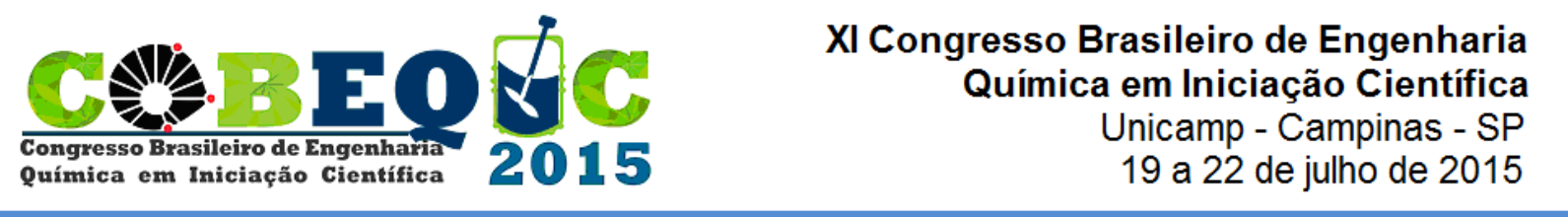

Figura 1. Mesocarpo de maracujá-amarelo in natura.

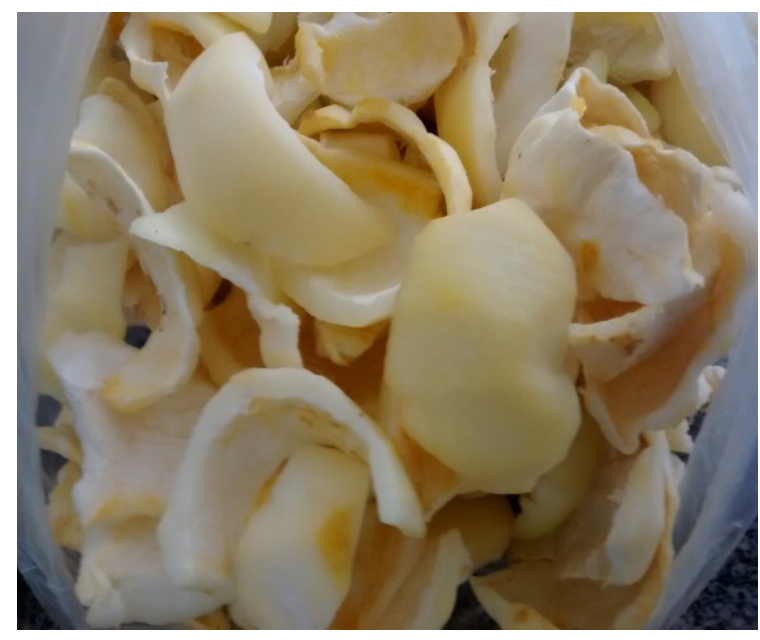

\subsection{Testes em Batelada de Remoção de Cr(VI)}

A solução estoque de $\mathrm{Cr}(\mathrm{VI})$ foi preparada a partir de dicromato de potássio dissolvendo $2,829 \mathrm{~g}$ em $1000 \mathrm{~mL}$ de água destilada, formando, assim, uma solução de 1000 $\mathrm{mg} \mathrm{L}^{-1}$ de $\mathrm{Cr}(\mathrm{VI})$. Foram obtidas, por diluições, soluções nas concentrações padrões de 0,2 ; 0,$4 ; 0,6 ; 0,8$ e $1,0 \mathrm{mg} \mathrm{L}^{-1}$ para determinação da curva de calibração do espectrofotometro, segundo procedimento de determinação de $\mathrm{Cr}(\mathrm{VI})$ por complexação com 1,5-difenilcarbazida em meio ácido (Morita e Assumpção, 2007). 0,1 g do biossorvente foi transferido para erlenmeyer juntamente com $100 \mathrm{~mL}$ da solução de $\mathrm{Cr}(\mathrm{VI}) 5 \mathrm{mg} \mathrm{L}^{-1} \mathrm{em} \mathrm{pH} \mathrm{2,} \mathrm{controlado} \mathrm{com}$ $\mathrm{HCl}$ 1,0 $\mathrm{mol} \mathrm{L}^{-1}$ e levados ao agitador (Nova Ética, mod. 109) a uma velocidade de agitação de $150 \mathrm{rpm}$. Foi transferida uma alíquota filtrada de $5 \mathrm{~mL}$ para balão volumétrico de $100 \mathrm{~mL}$, juntamente com $25 \mathrm{~mL}$ de ácido sulfúrico $0,1 \mathrm{~mol} \mathrm{~L}^{-1}$ e $2 \mathrm{~mL}$ de 1,5-difenilcarbazida $0,5 \mathrm{~g} \mathrm{~L}$ 1, completando o volume com água destilada. Posteriormente, a amostra foi analisada em espectrofotômetro (SP-220 da Biospectro) no comprimento de onda de $540 \mathrm{~nm}$, correspondente à faixa de $\operatorname{Cr}(\mathrm{VI})$ no espectro de emissão e a absorbância da solução foi verificada em triplicata. Todos os experimentos foram conduzidos à temperatura ambiente, em erlenmeyers cobertos e as leituras de $\mathrm{pH}$ foram feitas com o $\mathrm{pH}$ metro (Lutron $\mathrm{pH}$ 206). A concentração de $\mathrm{Cr}(\mathrm{VI})$ após a adsorção foi determinada usando o valor da absorbância da amostra. A porcentagem de cromo hexavalente removida foi determinada pela Equação 4.

$$
\% \text { Remoção de } \operatorname{Cr}(V I)=\left(\frac{C_{i}-C_{f}}{C_{i}}\right) \times 100
$$

\subsection{Equilíbrio de Biossorção}

Ao avaliar o equilíbrio químico de biossorção, foram realizados testes de remoção com concentração de $\mathrm{Cr}(\mathrm{VI})$ variando de 1 a $20 \mathrm{mg} \mathrm{L}^{-1}$, mantendo-se constante a massa do biossorvente. Os testes foram realizados segundo procedimento da seção 2.2 considerando as concentrações da solução de $\mathrm{Cr}(\mathrm{VI})$ citadas nesta seção e tempo de agitação de $8 \mathrm{~h}$. 


\section{RESULTADOS E DISCUSSÃO}

\subsection{Estudo do Equilíbrio Químico}

Os dados experimentais obtidos nas análises do equilíbrio de biossorção, apresentados na Figura 2, foram ajustados conforme os modelos de Langmuir e Freundlich, de acordo com as Equações 1 e 3, respectivamente. A forma da curva da Figura 2 é caracterizada por um decréscimo na inclinação da curva à medida que os sítios disponíveis para adsorção vão diminuindo, devido ao recobrimento da superfície adsorvedora, e indica que em baixas concentrações a superfície tem alta afinidade pela substância adsorvida.

Figura 2. Dados de equilíbrio químico para biossorção de Cr(VI) sobre mesocarpo de maracujá.

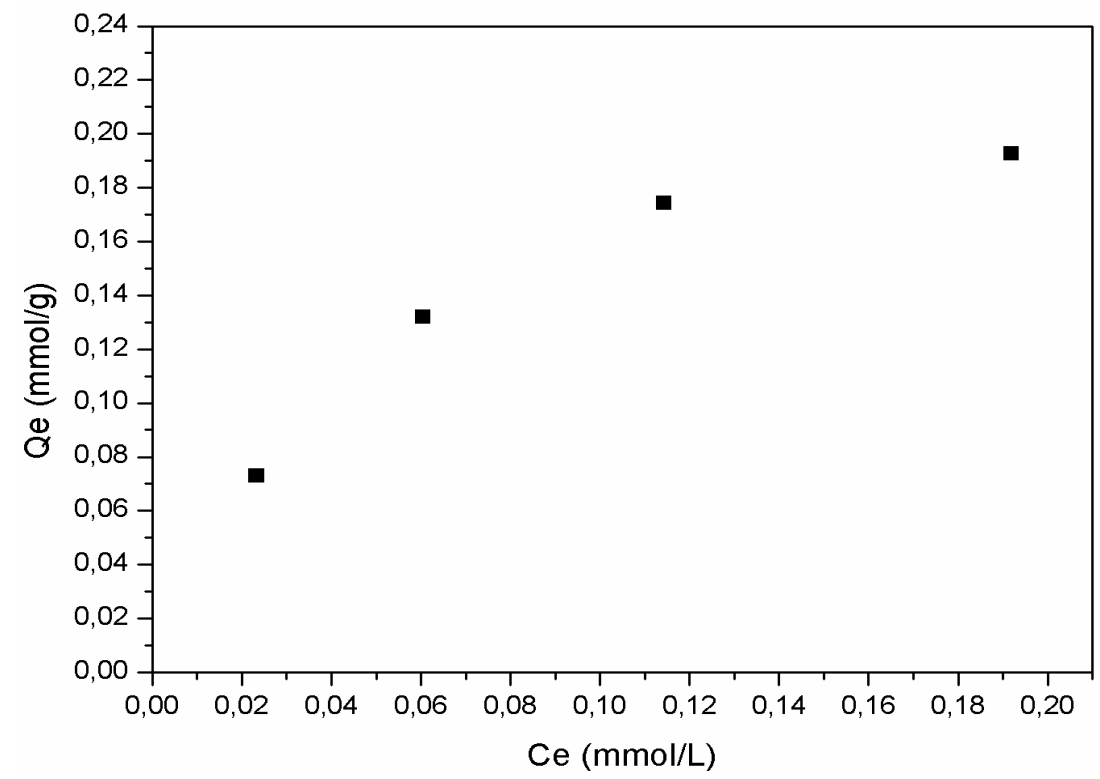

Os valores dos parâmetros de biossorção para as isotermas de Langmuir e Freundlich, mostrados na Tabela 1, foram estimados através de Programação Matemática Não-Linear, usando o software GAMS - General Algebraic Modelling System. A função objetivo utilizada foi a MPSED - The Marquardt's percent standard deviation, representada pela Equação 5, em que $\mathrm{Q}_{\mathrm{e} \text {,calc }}$ é valor o predito pelas isotermas; $\mathrm{Q}_{\mathrm{e}, \text { exp }}$ é o valor encontrado a partir dos experimentos; $\mathrm{N}$ é o número de dados obtidos; e $\mathrm{P}$ é o número de parâmetros de cada isoterma.

$$
\operatorname{MPSED}=\sqrt{\sum_{i=1}^{n} \frac{[(Q e, \exp -Q e, c a l) / Q e, \exp ]_{i}^{2}}{N-P}} \times 100
$$

Utilizou-se também o teste estatístico Qui-Quadrado para determinar a isoterma ótima para o processo de biossorção estudado. A vantagem de utilizar o teste Qui Quadrado, representado pela Equação 6, é comparar as duas isotermas na mesma abscissa e ordenada. Se o valor do modelo for similar ao valor experimental, $\chi^{2}$ deve ser um número pequeno e viceversa. 


$$
\chi^{2}=\sum \frac{\left(Q_{e, \exp }-Q_{e, \text { calc }}\right)^{2}}{Q_{e, \text { calc }}}
$$

Tabela 1. Determinação dos parâmetros das isotermas de Langmuir e Freundlich através da minimização da função erro MPSED e valor do $\chi^{2}$.

\begin{tabular}{cccccccc}
\hline Isoterma & $\mathrm{Q}_{\max }\left(\mathrm{mmol} \mathrm{g}^{-1}\right)$ & $\mathrm{k}_{\mathrm{L}}\left(\mathrm{L} \mathrm{g}^{-1}\right)$ & $\mathrm{R}_{\mathrm{L}}$ & $\mathrm{k}_{\mathrm{F}}\left(\mathrm{L} \mathrm{mmol}^{-1}\right)$ & $\mathrm{n}$ & $\mathrm{FO}^{*}$ & $\chi^{2}$ \\
\hline Langmuir & 0,257 & 17,183 & 0,131 & -- & -- & 5,909 & 0,001 \\
\hline Freundlich & -- & -- & -- & 0,478 & 2,046 & 5,422 & $7,8318 \times 10^{-4}$ \\
\hline
\end{tabular}

*Valor da função objetivo encontrado.

Uma vez que o valor de $\chi^{2}$ foi menor para a isoterma de Freundlich, este modelo descreve melhor a biossorção de Cr(VI) sobre o biossorvente. Pode-se inferir, então, que a superfície do mesocarpo de maracujá utilizado neste estudo é heterogênea. $\mathrm{O}$ valor de $\mathrm{n}$ está no intervalo de 0 a 10 , indicando um comportamento favorável à biossorção de $\mathrm{Cr}(\mathrm{VI})$. A constante $\mathrm{k}_{\mathrm{F}}$ é uma medida aproximada da capacidade de adsorção do adsorvente; quanto maior o seu valor, maior é a capacidade de adsorção. No entanto, $\mathrm{k}_{\mathrm{F}}$ não fornece a capacidade máxima de biossorção, como no caso da constante $Q_{\max }$ de Langmuir, uma vez que o modelo de Freundlich não prevê a saturação do biossorvente.

Comparando o valor obtido no presente estudo para a capacidade de biossorção máxima do biossorvente $\left(\mathrm{Q}_{\max }\right)$ com valores reportados na literatura, mostrados na Tabela 2, pode-se perceber que o mesocarpo de maracujá é o que apresenta maior capacidade de biossorção, podendo adsorver até $13 \mathrm{mg}$ para cada $1 \mathrm{~g}$ de $\mathrm{Cr}(\mathrm{VI})$ presente em solução.

Tabela 2. Comparação entre as capacidades máximas de biossorção de alguns biossorventes para o $\mathrm{Cr}(\mathrm{VI})$

\begin{tabular}{cccccc}
\hline Biossorvente & $\mathrm{Q}_{\max }\left(\mathrm{mg} \mathrm{g}^{-1}\right)$ & $\begin{array}{c}\text { Tempo de } \\
\text { Equilíbrio }\end{array}$ & $\mathrm{pH}$ & Massa (g) & Ref \\
\hline $\begin{array}{c}\text { Serragem de Carvalho } \\
\text { Modificada }\end{array}$ & 1,7 & $8 \mathrm{~h}$ & 3,0 & 60 & {$[1]$} \\
\hline Areia Modificada & 1,19 & $1 \mathrm{~h} 20$ & 2,5 & -- & {$[2]$} \\
\hline Nano- $\mathrm{Al}_{2} \mathrm{O}_{3}$ & 8,56 & $1 \mathrm{~h}$ & 2,0 & -- & {$[3]$} \\
\hline Casca de Macaxeira & 6,838 & $120 \mathrm{~min}$ & 2,0 & -- & {$[4]$} \\
\hline Mesocarpo de Coco Seco* $^{*}$ & 10,738 & $160 \mathrm{~min}$ & 2,0 & -- & {$[4]$} \\
\hline Mesocarpo de Maracujä $^{*}$ & 13,0 & $3 \mathrm{~h}$ & 2,0 & 0,1 & $\begin{array}{c}\text { Presente } \\
\text { Estudo }\end{array}$ \\
\hline
\end{tabular}

* com tratamento térmico

\section{CONSIDERAÇÕES FINAIS}

A maior remoção de $\mathrm{Cr}$ foi de $75 \%$ utilizando $0,1 \mathrm{~g}$ de adsorvente (100 mesh) com pH inicial 2,0. Os dados de adsorção ajustaram-se melhor ao modelo de isoterma de Freundlich, indicando que a superfície do biossorvente utilizado é heterogênea. A capacidade de biossorção máxima, segundo modelo de Langmuir, foi de $13 \mathrm{mg}$ de $\mathrm{Cr}$ (VI) para cada grama do biomaterial. Os resultados indicaram boas perspectivas na aplicação do mesocarpo de 


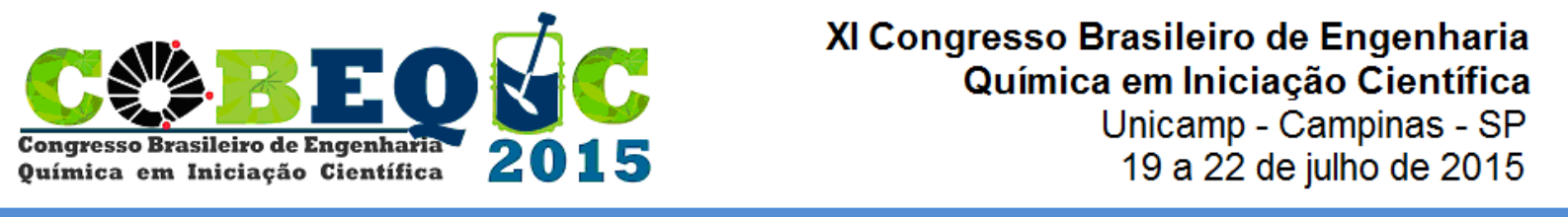

maracujá, um resíduo da agroindústria, como um adsorvente alternativo e economicamente favorável na remoção de $\mathrm{Cr}(\mathrm{VI})$ de efluentes industriais.

\section{REFERÊNCIAS BIBLIOGRÁFICAS}

[1] ARGUN, M. E.; DURSUN, S.; OZDEMIR, C.; KARATAS, M. Heavy metal adsorption by modified oak sawdust: thermodynamics and kinetics. J. Hazard. Mater., v. 141, p. 77-85, 2007.

[2] YADAV, S.; SRIVASTAVA, V.; BANERJEE, S.; WENG, C.H.; SHARMA, Y. C. Adsorption characteristics of modified sand for the removal of hexavalent chromium ions from aqueous solutions: kinect, thermodynamic and equilibrium studies. Catena; v. 100, p. 120-127, 2013.

[3] SHARMA, Y. C.; SRIVASTAVA, V.; MUKHERJEE A. K. Synthesis and application of Nano- $\mathrm{Al}_{2} \mathrm{O}_{3}$ poder of for the reclamation of hexavalent chromium from aqueous solution. J. Chem. Eng., v. 55, p. 2390-2398.

[4] PEIXOTO, P. do N.; Potencialidade de remoção de $\mathrm{Cr}(\mathrm{VI})$ de soluções aquosas por biossorção usando resíduos da agroindústria. Tese (Mestrado em Engenharia Química), Universidade Federal de Sergipe, Sergipe, Brasil, 2015.

CONCEIÇÃO, J. do C.; RAMOS, V. H. S.; SANTOS, E. de J.; SILVA, A. S.; COSTA, A. W. M. de C. Biosorption $\mathrm{f} \mathrm{Cr}(\mathrm{VI})$ from aqueous solutions using chemically modified okra powder. J. of Basic \& Applied Sciences, v. 10, p. 73-79, 2014.

MORITA, T.; ASSUMPÇÃO, R. M. V. Manual de soluções, reagentes e solventes: padronização, preparação, purificação com indicadores de segurança e de descarte de produtos químicos. São Paulo: Editora Blucher, 2007.

PINA, F. D. S. Tratamento de águas contaminadas com crómio(VI) por bioadsorção em algas marinhas. 65f. Tese (Mestre em Engenharia do Ambiente), Faculdade de Engenharia da Universidade do Porto, Porto, Portugal, 2011. 